\title{
High-content imaging of neutral lipid droplets with 1,6-diphenylhexatriene
}

\author{
Max V. Ranall, Brian G. Gabrielli, and Thomas J. Gonda \\ University of Queensland Diamantina Institute, Princess Alexandra Hospital, Brisbane QLD, Australia
}

BioTechniques 50: 35-42 (July 2011) doi 10.2144/000113702

Keywords: high-content imaging; high-throughput screening; lipid droplet; 1,6-diphenyl-1,3,5-hexatriene; DPH; Nile Red; BODIPY 493/503

Neutral lipid droplets (LDs) are dynamic lipid storage organelles found in all eukaryotic cells from yeast to mammals and higher plants. LDs are important to many physiological processes that include basic cellular maintenance, metabolism, and diverse medical pathologies. LD accumulation has been studied extensively by a range of methods, but particularly by microscopy with several fluorescent dyes extensively used for qualitative and quantitative imaging. Here, we compared established LD stains Nile Red and BODIPY 493/503 to the 4',6-diamidino-2-phenylindole (DAPI)-range dye 1,6-diphenyl-1,3,5-hexatriene (DPH; excitation/emission $\lambda_{\max }=350 \mathrm{~nm} / 420 \mathrm{~nm}$ ) using high-content image analysis. HeLa cells treated with oleic acid or vehicle were used to compare staining patterns between DPH and Nile Red as well as DPH and the LD protein adipophilin. DPH, Nile Red, and BODIPY 493/503 were compared as assay reagents in oleic acid dose-response experiments. Treatment of MCF-7 cells with sodium butyrate was used as a second cellular system for high-content analysis of LD formation. In this experimental context, we demonstrate the compatibility of DPH with GFP, a technical limitation of Nile Red and BODIPY 493/503 dyes. These data show that DPH has comparable sensitivity and specificity to that of Nile Red. Z'-factor analysis of dose-response experiments indicated that DPH and BODIPY 493/503 are well suited for quantitative analysis of LDs for high-throughput screening (HTS) applications.

Lipid droplets (LDs) are ubiquitous intracellular structures whose formation, growth, and maintenance are highly regulated $(1,2)$. Lipid metabolism and droplet dynamics are of considerable interest to agriculture, biofuel production, viral pathology, nutrition, and cancer biology $(3,4)$. Physiological dysregulation of fatty acid homeostasis can result in diverse clinical pathologies including type 2 diabetes $(5,6)$. Further, LDs are intimately involved with the intracellular synthesis of sterols and serve as the reservoir of essential raw materials for biological membrane structures (7). Microscopy has been an indispensable tool for describing LD biology with various stains applied in histology (8), fluorescent imaging $(9,10)$, genetic screens (11-14), and high-content imaging (13-18).

Nile Red is perhaps the most common fluorescent LD stain and exhibits robust fluorescence in the nonpolar environment of lipids $(9,19)$. This dye exhibits variable excitation and emission properties depending on solution polarity (20), and this fluorescence spectral shift has proven useful distinguishing different intracellular hydrophobic structures $(21,22)$. Nile Red-stained LDs excited in the fluorescein isothiocyanate (FITC) channel have a yellowgold fluorescence emission $\left(\lambda_{\max }=530\right.$ $\mathrm{nm}$ ) that can be readily observed with a wide-pass green (FITC) emission filter $(9,19,20)$, while membrane bilayers are more efficiently detected in the orange (tetramethylrhodamine5 - is othiocyanate/tetramethylrhodamine-6-isothiocyanate; TRITC) or red (Texas Red) fluorescence channels $(9,21,22)$. The large spectral occupancy of Nile Red complicates its use with other fluorophores in the FITC, TRITC, and Texas Red ranges (9). BODIPY 493/503 is a FITC-range dye with greater LD specificity and a smaller fluorescent spectral footprint than Nile Red (9,23). BODIPY 493/503, however, has some aberrant fluorescent properties that must be considered prior to multicolor fluorescent analysis (24). Recovering the green channel is of considerable interest, because GFP is the most widely used fluorescent protein for transcriptional and translational fusions, while FITC-range dyes are among the most sensitive and robust fluorescent probes. The near-red BODIPY-family dye LD540 recovers the FITC channel and shows excellent specificity for labeling LDs (25) but is not commercially available. The red and deep red LipidTOX dyes (Invitrogen, Carlsbad, CA, USA) are FITC-compatible and available at considerable expense. The 4',6-diamidino-2-phenylindole (DAPI)range fluorophore 1,6-diphenyl-1,3,5hexatriene $(\mathrm{DPH})$ is only fluorescent in hydrophobic environments and was originally applied in the characterization of biological membranes (26). This dye was subsequently identified as an LD stain for use with fluorescent microscopy 


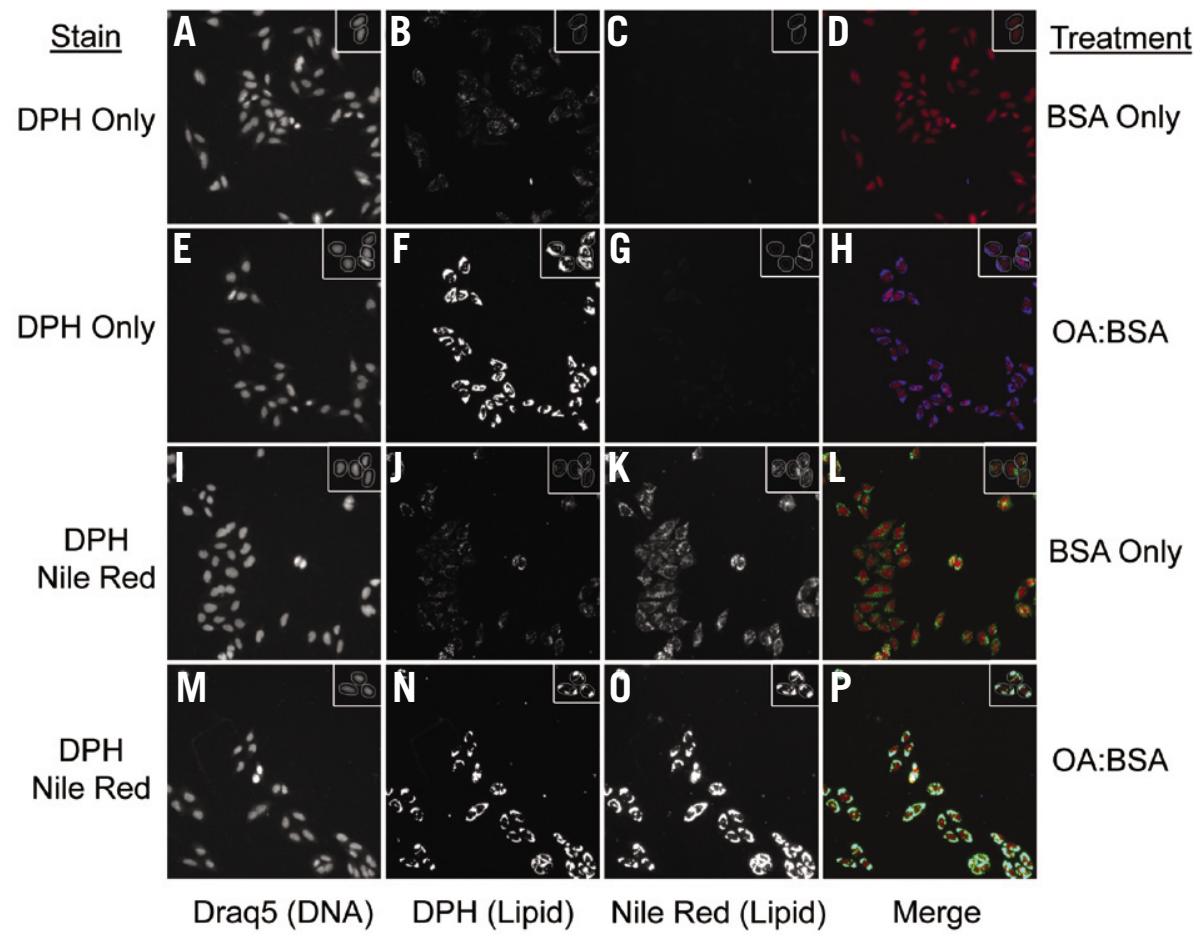

Figure 1. DPH and Nile Red staining of OA-treated HeLa cells. HeLa cells seeded in 384-well plates were treated overnight with BSA-only vehicle control $(A-D$ and $I-L)$ or with OA-loaded BSA (E-H and M-P). Cells were fixed with formaldehyde and stained with $2 \mu \mathrm{M}$ Draq5 nucleic acid stain to visualize nuclei ( $\mathrm{A}, \mathrm{E}, \mathrm{I}$, and $\mathrm{M}$ ) and $4 \mu \mathrm{M}$ DPH (B, F, J, and $\mathrm{N}$ ) in the absence (C and G) or presence (K and O) of $100 \mathrm{ng} / \mathrm{mL} \mathrm{Nile} \mathrm{Red.} \mathrm{Inset}$ panels in the top right corner of each single-channel image indicate ROIs used for quantitative analysis of staining. Pseudocolored composite images ( $,, H, L$, and $P$ ) approximately correspond to the emission colors of each fluorophore with Draq5 in red, DPH in blue, and Nile Red in green. Single-channel images were acquired at 20x magnification through a Pinkel filter set (multi-bandpass emission filter) individually exciting Draq5 with the Cy5 filter, DPH with the DAPI filter, and Nile Red with the FITC filter.

(27), but has been underutilized perhaps because it shares excitation and emission properties with the widely used DAPI and Hoechst DNA stains.

Here we report the efficacy and specificity of DPH for analysis of LDs by high-content imaging using previously described reagents and treatments. We compared DPH staining with that of Nile Red and the LD-specific protein adipophilin (28). We compared DPH, Nile Red, and BODIPY 493/503 as highcontent screening reagents in oleic acid (OA) dose-response treatments of $\mathrm{HeLa}$ cells. Additionally, we quantified LD formation in GFP-expressing MCF-7 cells in sodium butyrate dose-response treatments. Assay resolution and highthroughput screening (HTS) suitability were estimated using $Z^{\prime}$-factor calculations (29). Together, the data presented here validate DPH to be a sensitive and effective reagent for high-content analysis of LDs with similar performance to Nile Red and BODIPY 493/503. In addition to being sensitive, specific, effective, and economical, DPH is also compatible with FITC-range fluorophores allowing novel high-content imaging strategies.

\section{Materials and methods}

Instrumentation

Liquid handling was performed with a SciClone ALH3000 (Caliper Life Sciences, Hopkinton, MA, USA), washing with an ELx405 plate washer (BioTek Instruments, Winooski, VT, USA), and bulk dispensing with a Wellmate (Thermo Fisher Scientific, Waltham, MA, USA).

High-content imaging was performed with a Cellomics Arrayscan Vti (Thermo Fisher Scientific) equipped with an $\mathrm{X}$-cite120 light source (ExFo, Mississauga, Ontario, Canada) and two different four-color DAPI/FITC/ TRITC/Cy5 fluorescence filter sets (Omega Optical, Brattleboro, VT, USA). Dose-response and DPH costaining experiments were imaged with a Pinkel set (multiple excitation filters, single multi-bandpass emission filter). Immunofluorescence and some Nile Red experiments were imaged with a Sedat set (multiple excitation filters, multiple single-bandpass emission filters) with excitation and emission filters for DAPI (ex/em: 365WB50/450DF65), FITC (ex/em: 485DF20/525WB20), TRITC (ex/em: 555DF10/585W B20), and Cy5 (ex/em: 640AF20/695AF55) imaging channels.

\section{Cell culture and treatments}

Unless otherwise noted, all reagents were obtained from Sigma-Aldrich (Castle Hill, NSW, Australia). HeLa cells were seeded at 750 cells/well in $25 \mu \mathrm{L}$ into black, clear-bottom 384-well tissue culture plates (part no. 3712; Corning, Lowell, MA, USA). OA was prepared as a complex with BSA (OA:BSA) at a molar ratio of 6:1 (9). A $100 \mathrm{mM}$ OA stock was prepared in $0.1 \mathrm{M} \mathrm{NaOH}$ at $70^{\circ} \mathrm{C}$. Cold alcohol isolated BSA (Biotechnology grade; AMRESCO, Solon, OH, USA) did not require delipidation; $3 \mathrm{~g}$ were dissolved with stirring in $\sim 20 \mathrm{~mL}$ water with $3 \mathrm{~mL}$ of $10 \times$ PBS. This solution was then split into equal volumes for OA:BSA and BSA-only solutions. OA:BSA was prepared by dropwise addition of the OA stock solution $(1.35 \mathrm{~mL})$ to stirring BSA solution at $50^{\circ} \mathrm{C}$. After $20 \mathrm{~min}$ of stirring at temperature, solution was allowed to cool to room temperature, $\mathrm{pH}$ adjusted to $\sim 7.4$ with $2 \mathrm{M} \mathrm{NaOH}$ 

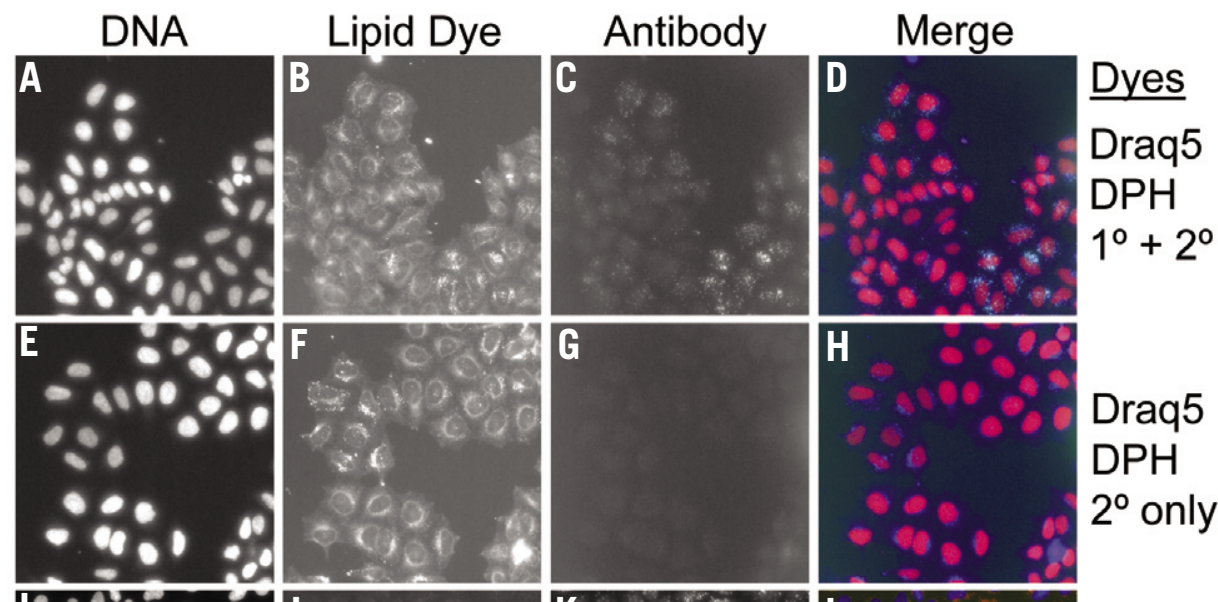

\section{Draq5 \\ $\mathrm{DPH}$ \\ $2^{\circ}$ only}
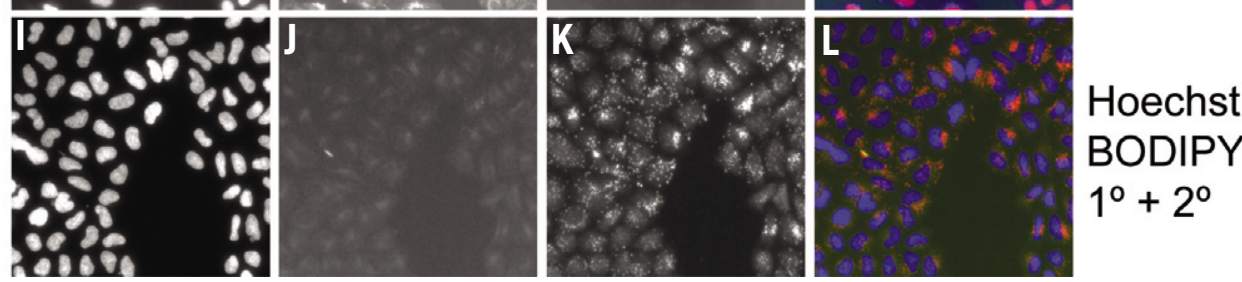

Figure 2. Adipophilin immunolabeling with DPH or BODIPY 493/503 staining of HeLa cells. HeLa cells seeded in 384-well plates were treated overnight with the BSA-only equivalent of $\sim 1 \mu \mathrm{M}$ OA treatment. Formaldehyde-fixed cells were labeled with mouse anti-adipophlilin primary antibody $\left(1^{\circ}\right)$ and Alexa Fluor 488 anti-mouse (C, green), Alexa Fluor 488 anti-mouse only (G, green), or Alexa Fluor 647 (K, red) secondary antibodies ( $2^{\circ}$ ). DNA was stained with $2 \mu \mathrm{M}$ Draq5 nucleic acid stain (A and E, red) or $5 \mu \mathrm{g} / \mathrm{mL}$ Hoechst 33342 (I, blue). LDs were stained with $2 \mu \mathrm{M}$ DPH (B and $\mathrm{F}$, blue) or with $1 \mu \mathrm{g} / \mathrm{mL}$ BODIPY 493/503 (J, green). Images were acquired with a 20x objective using a DAPI/FITC/TRITC/Cy5 Sedat filter set.

(usually $<200 \mu \mathrm{L}$ ), volume to $15 \mathrm{~mL}$ with water, and filter-sterilized. The BSA-only solution was prepared identically to the OA:BSA solution except the $0.1 \mathrm{M}$ $\mathrm{NaOH}$ contained no OA. BSA-only or OA:BSA treatments were prepared as a 2 -fold dilution series in complete media. A day after seeding, $8 \mu \mathrm{L}$ of the dilution series were mixed into each well giving final well volumes of $33 \mu \mathrm{L}$ and $\mathrm{OA}$ concentrations from $\sim 1$ to $\sim 1000 \mu \mathrm{M}$. Cells were treated overnight, washed with PBS, fixed with 3.7\% formaldehyde in PBS for 20 min at room temperature, washed again with PBS, and then stored at $4^{\circ} \mathrm{C}$ for up to 7 days.

MCF-7 cells were seeded at 2000 cells/well in $100 \mu \mathrm{L}$ into black, clearbottomed 96-well plates (Viewplate; Perkin Elmer, Waltham, MA, USA). A day after seeding, $16 \mathrm{mM}$ sodium butyrate was prepared in complete medium, filter-sterilized, and dilutions added to wells as a $2 \times$ concentrate in $100 \mu \mathrm{L}$. Cells were treated for $72 \mathrm{~h}$, then washed and fixed as above.

Stain preparation and application Nile Red (Sigma-Aldrich) stock solution was $1 \mathrm{mg} / \mathrm{mL}$ in acetone; a $100 \mu \mathrm{g} / \mathrm{mL}$ intermediate acetone dilution was used to prepare $100 \mathrm{ng} / \mathrm{mL}$ Nile Red in PBS with $5 \mu \mathrm{g} / \mathrm{mL}$ Hoechst 33342. BODIPY
493/503 (1 mg/mL; Invitrogen) was provided by Rob Parton (University of Queensland, St. Lucia, Australia) and applied at $1 \mu \mathrm{g} / \mathrm{mL}$ in PBS with $5 \mu \mathrm{g} / \mathrm{mL}$ Hoechst 33342. DPH (Sigma-Aldrich) was dissolved in DMSO at $2 \mathrm{mM}$, mixing by inversion for $\sim 1 \mathrm{~h}$ at room temperature in the dark. This stock was added dropwise to vigorously mixing PBS to $2-4 \mu \mathrm{M}$ at room temperature in the dark, allowing the aqueous DPH dispersion to mix $(27,30)$ before addition of Draq5 (31) (Biostatus Ltd., Shepshed, Leicestershire, $\mathrm{UK})$ to $2 \mu \mathrm{M}$ and application to samples. Preliminary data reflected effective DPH staining when a $200 \mu \mathrm{M}$ DMSO stock was diluted into a blocking-permeabilization solution (see below) with minimal mixing. Solutions for DPH/ Nile Red costaining experiments were prepared as DPH solutions above with Draq 5 as the nucleic acid stain instead of Hoechst. Controls for fluorescent channel bleed through were stained with only Nile Red or DPH in both BSA-only and OA:BSA treatment groups. Prior to staining, plates were allowed to warm to room temperature, and wells were emptied of PBS into a sink immediately before applying $40 \mu \mathrm{L} /$ well of the staining solutions with either a liquid handling robot or a bulk dispenser. All plates were sealed with black sealing film
(Perkin Elmer) and incubated at room temperature overnight before imaging. BODIPY-stained samples had to be washed with PBS prior to imaging.

Immunofluorescence

BSA-only or OA:BSA-treated HeLa cells were immunostained for adipophilin as previously described (9) in a blocking-permeabilization solution: $3 \%$ BSA (w/v) and $0.1 \%(\mathrm{w} / \mathrm{v})$ saponin in PBS. Mouse anti-adipophilin (clone AP125; Progen, Heidelberg, Germany; provided by Rob Parton) was diluted 1:3 $(\mathrm{v} / \mathrm{v})$ in the blocking-permeabilization buffer; rabbit polyclonal anti- $\alpha$-tubulin (AbCam, Cambridge, MA, USA), antirabbit AlexaFluor 488 (Invitrogen), and anti-mouse AlexaFluor 555 (Invitrogen) were used at 1:500. Control-stained cells of both BSA-only and OA:BSA treatments received no primary antibody but were stained with secondary antibodies, Draq 5 and DPH. Identically treated cells were also stained with BODIPY 493/503 and Hoechst 33342 as above, except using anti-mouse AlexaFluor 647 and anti-rabbit AlexaFluor 555 secondary antibodies.

High-content imaging and analysis Image acquisition and analysis were controlled with the Cellomics Arrayscan 


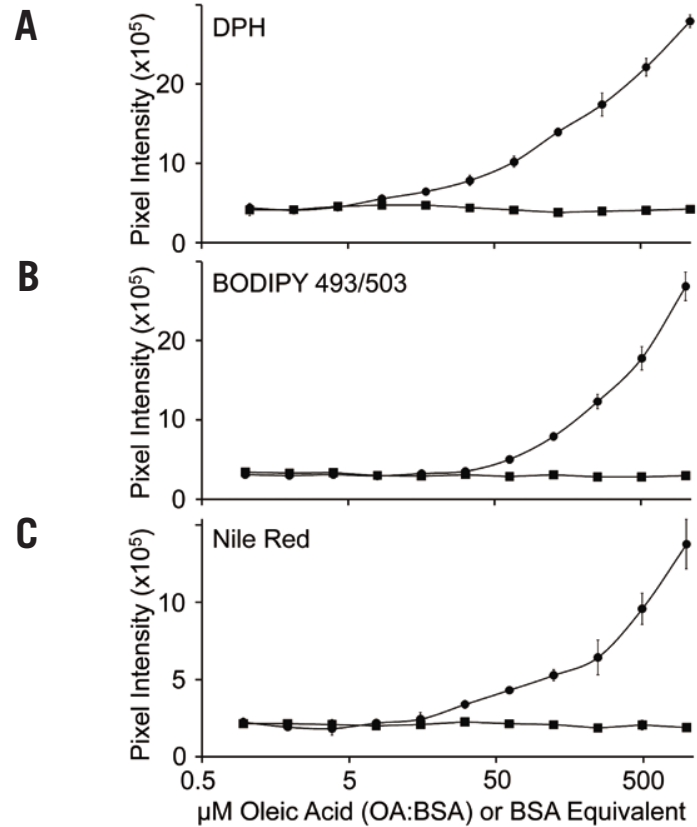

Figure 3. Quantitative fluorescent microscopy of OA-treated HeLa cells stained with DPH, BODIPY $493 / 503$, or Nile Red. HeLa cells seeded in 384-well plates were treated overnight with a 2-fold dilution series of OA-loaded BSA $(\bullet)$ or with an equivalent dilution series of BSA-only ( $\mathbf{\square})$. Cells were fixed with formaldehyde and stained overnight with $2 \mu \mathrm{M}$ Draq5 nucleic acid stain to identify nuclei and $4 \mu \mathrm{M}$ DPH (A) or $5 \mu \mathrm{g} / \mathrm{mL}$ Hoechst 33342 and $1 \mu \mathrm{g} / \mathrm{mL}$ BODIPY 493/503 (B) or $100 \mathrm{ng} / \mathrm{mL}$ Nile Red (C). Nucleic acid stains were used to delimit the edge of the nucleus, and the total lipid dye pixel intensity was collected $\sim 7 \mu \mathrm{m}$ beyond the nuclear boundary as indicated in the single-channel insets in Figure 1. Data points are the mean value from four replicate wells, sampling at least 200 cells/well; error bars equal 95\% confidence interval (CI).

Compartmental Analysis (v.3) application software, maintaining equivalent acquisition and processing parameters within each experiment. All exposure times were adjusted empirically to $<80 \%$ of pixel saturation in positive control reference wells not included in the experimental data set. Nuclei were imaged in the Cy5 channel for Draq5stained samples or the DAPI channel for Hoechst-stained samples, Nile Red staining was imaged in FITC and TRITC channels, DPH was imaged in the DAPI channel, while BODIPY was imaged in the FITC channel. Nuclei were identified using a fixed pixel-intensity threshold, adjusting segmentation parameters and exposure times manually in reference wells included in each experiment. DPH, Nile Red, or BODIPY stain total pixel intensities were collected within the region of interest (ROI) extending $\sim 7 \mu \mathrm{m}$ beyond the nuclear perimeter (see Figure 1, insets). For all channels, local background correction was applied, by the surface fitting method, using a radius of $40-50 \mu \mathrm{m}$ beyond the nuclear perimeter or extranuclear ROI. Mean total pixel intensity in the ROI was measured from at least 200 cells/well for
HeLa cells and 16 fields for MCF-7 cells with 4 replicate wells/treatment.

The staining methods described here were evaluated for HTS assay suitability with the $Z^{\prime}$-factor calculation (29). This metric compares the experimental treatment, either OA or butyrate $(+)$, to the corresponding vehicle control (-). The mean ROI total pixel intensity from replicate wells was used to calculate sample mean $(\mu)$ and standard deviation $(\sigma)$ and $Z^{\prime}$-factor calculated as described previously (29) to give $Z^{\prime}=1-\left(3\left[\sigma^{(+)}+\sigma^{(-)}\right] /\left[\mu^{(+)}-\mu^{(-)}\right]\right)$. This metric quantifies the separation band between the experimental and vehicle control treatments, while remaining sensitive to intratreatment variation. Data that yielded $0.1<Z^{\prime}<0.5$ reflects "good" assay suitability for HTS, while $0.5<Z^{\prime}<$ 1 reflects "excellent" assay suitability.

\section{Results and discussion}

To test DPH as a dye for labeling neutral LDs, we compared DPH with the established and structurally unrelated LD-stain Nile Red in HeLa cells treated with OA. Cells were treated overnight with BSA-only (Figure 1, A-D and I-L) or OA complexed to BSA (OA:BSA; Figure

\section{The Evolution of Pipetting Xcellence Continues}

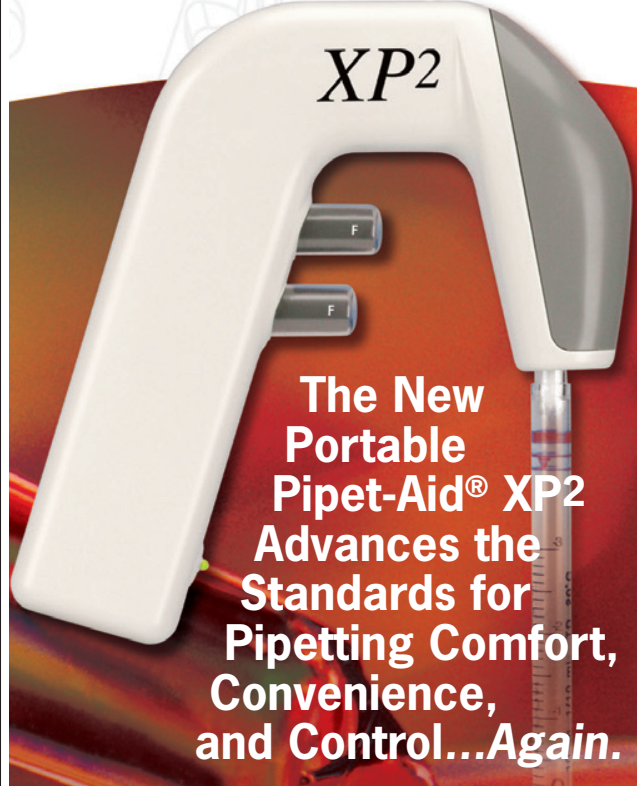

- New Ergonomic Design-The most comfortable pipettor you ever laid a hand on

- New Power Source for Uninterrupted Extended Operation-Can be charged while in use

- New Ultra Quiet Precision PumpGreat control for aspiration or dispensing

Building on the years of experience of providing state-of-the-art tools that make your job in the laboratory safer, easier, and more convenient, the Pipet-Aid XP2 delivers more. The ergonomic design is more comfortable to handle. The new power source enables more convenient extended operation and the new more powerful pump provides great control and quieter operations. The unit is supplied complete with a power/supply charger, 4 extra filters, and a stand to enable it to be set down.

For a copy of our new catalog or more information, call 800/523-7480 or visit our website at drummondsci.com

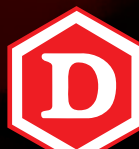
DRUMMOND SCIENTIFIC COMPANY

500 Parkway, Box 700 Broomall, PA 19008

The Developers of 


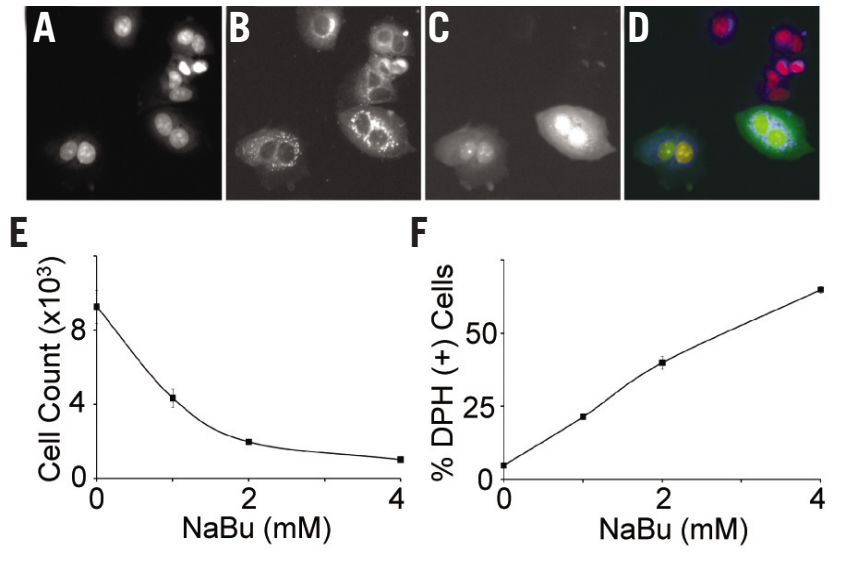

Figure 4. DPH staining of GFP-expressing MCF-7 cells treated with sodium butyrate. MCF-7 cells were seeded in 96 -well plates and treated for $72 \mathrm{~h}$ with sodium butyrate or vehicle control. Cells were fixed with formaldehyde and then stained with $2 \mu \mathrm{M}$ Draq5 and $4 \mu \mathrm{M} \mathrm{DPH}$. Draq5 (A), DPH (B), and GFP (C) were respectively imaged with Cy5, DAPI, and GFP excitation filters and pseudocolored red, blue, and green in the composite image (D). Mean cell counts from equivalent acquisition fields in four wells following $72 \mathrm{~h}$ treatment with 0,1 , 2, or $4 \mathrm{mM}$ sodium butyrate (E). As above, total DPH fluorescence was collected on a per cell basis within a 7- $\mu \mathrm{m}$ radius of the nuclear perimeter. A DPH bright threshold was selected to quantify the fraction of the MCF-7 cell population with a strong LD staining following experimental sodium butyrate treatment $(F)$. Each data point is the mean value from four replicate wells in which an equivalent number of fields were imaged with a $10 \times$ objective; error bars equal $95 \% \mathrm{Cl}$. $\mathrm{NaBu}$, sodium butyrate.

$1, \mathrm{E}-\mathrm{H}$ and $\mathrm{M}-\mathrm{P})$. Parallel treatments were stained with DPH only (Figure 1, $\mathrm{A}-\mathrm{H}$ ) or DPH and Nile Red (Figure 1, I-P). To visualize nuclei in the presence of DPH - a blue-emitting fluorophore similar to DAPI and Hoechst nucleic acid stains-DNA was stained with the far-red nucleic acid stain Draq5 (31) (Figure 1, A, E, I, and M). Cells treated with OA:BSA stained intensely with DPH (Figure 1, F and N) and Nile Red (Figure 1O). By comparison, BSA-only treatments exhibited less intense staining with DPH (Figure 1, B and J) and Nile Red (Figure 1K). Costaining OA:BStreated cells with both DPH and Nile Red gave overlapping staining patterns (Figure 1P). Notably, samples stained with DPH alone showed essentially no fluorescence in the FITC channel (Figure $1, C$ and $G$ ), while samples stained with Nile Red alone did not show detectable fluorescence in the DAPI channel (data not shown). Both DPH and Nile Red fluoresce in hydrophobic environments, but are quenched in aqueous solutions allowing samples to be imaged directly in the staining solution. An identical experiment conducted with DPH and BODIPY 493/503 gave similar results but had to be washed prior to imaging (data not shown). In the absence of largescale LD formation-such as in the BSA-only treatments (Figure 1, A-D and I-L, and Figure 2)-DPH and Nile
Red (especially in TRITC channel) can be used as a whole-cell stains.

To test the specificity of DPH for LDs, cells were immunolabeled for adipophilin and then stained with either DPH or BODIPY 493/503. Adipophilin stably accumulates on the surface of LDs, but is otherwise rapidly degraded and is a reliable LD marker $(10,28)$. HeLa cells used for this immunofluorescence analysis were treated overnight with the BSA-only vehicle equivalent to the lowest (1 $\mu \mathrm{M}$ OA) OA:BSA treatment. In these cells, DPH membrane fluorescence is visible with bright puncta present in many of the cells (Figure 2, B and F). These puncta exhibit specific adipophilin immunoreactivity (Figure 2C) that is absent in control cells prepared identically except without the adipophilin primary antibody (Figure 2G). An alternative imaging strategy of identically treated cells used Hoechst, BODIPY 493/503, and AlexaFluor 647 secondary antibody (Figure 2, I-L). This approach presented cleaner adipophilin labeling but BODIPY failed to specifically label the discrete LD puncta as imaged here. In cells loaded with $\sim 500 \mu \mathrm{M}$ OA, both DPH and BODIPY showed similar staining patterns to adipophilin immunofluorescence (data not shown).

To evaluate DPH as an assay reagent for high-content analysis and HTS, we conducted OA dose-response treatments on HeLa cells. Parallel 2-fold dilutions of OA:BSA or BSA-only vehicle control were applied to give final OA concentrations of $\sim 1$ to $\sim 1000 \mu \mathrm{M}$. Fixed cells were stained with DPH (Figure 3A), BODIPY 493/503 (Figure 3B), or Nile Red (Figure $3 C)$. Treatments were analyzed by highcontent imaging, quantifying total pixel intensity within an ROI extending $\sim 7$ $\mu \mathrm{m}$ beyond the nuclear perimeter (Figure 1 , inset of single channel images). This type of data analysis is not platformspecific and can be performed with most high-content analysis software. The Cellomics Arrayscan spot detector did provide superior assay performance, but those data were not presented here, as the spot detector is specialized software. Nile Red-stained cells were imaged in both FITC and TRITC channels using single-bandpass emission filters (Sedat optics). Nile Red fluoresced brighter in the TRITC channel than in FITC, and both channels detected LD formation with increasing OA concentration. With equivalent pixel saturation levels at highest $O A$ concentrations, the FITC channel exhibited superior assay dynamic range between BSA-only and OA:BSA treatments (data not shown). This observation is consistent with the previous reports that Nile Red has an emission $\lambda_{\max }$ of $\sim 530 \mathrm{~nm}$ in nonpolar mediums (20) and that Nile Red stained LDs exhibit yellow-gold fluorescence (19) best detected in the FITC channel (9). The dose-response series imaged with DPH (Figure 3A) closely resembled that of Nile Red (Figure 3C), while the series imaged with the more $\mathrm{LD}$-specific BODIPY 493/503 (Figure 3B) appears to be an almost ideal dose-response curve.

In a different cell context, LD formation was induced by treating MCF-7 cells with sodium butyrate $(32,33)$. Cells were treated with a doseresponse series, fixed, then stained with Draq5 (Figure 4A) and DPH (Figure 4B). These cells were stably transformed with GFP, although GFP-dim and GFP-null cells arise at some frequency (Figure 4C). Throughout these experiments, DPH did not interfere with the detection of the GFP-null and GFP-dim cells (Figure $4 \mathrm{C}$ and data not shown). As previously described (32-34), butyrate treatment is cytotoxic with an LD50 of $\sim 1 \mathrm{mM}$ (Figure 4E). In the surviving cells, total DPH fluorescence in the ROI increased substantially. Since the LD phenotype was not penetrant in the entire surviving cell population, an arbitrary DPH bright threshold was established such that 2\% of untreated cells were positive. Accord- 
ingly, the fraction of DPH bright cells increased with butyrate concentration (Figure 4F), consistent with surviving cells exhibiting increased LD formation (33). Parallel MCF-7 treatments were stained with Nile Red or LipidTOX deep red. Imaging with these reagents yielded LD formation data nearly identical to DPH (data not shown).

The LipidTOX reagent tested here exhibited similar sensitivity and assay resolution to both Nile Red and DPH (data not shown). Like DPH, this reagent was FITC-compatible and did not interfere with identification of GFP-dim and GFP-null cells (data not shown). LipidTOX deep red did not, however, exhibit particularly good stability with performance of the dye decomposing prior to conducting the HeLa experiments described above. By contrast, DPH stock solutions are extremely stable; an $\sim 4 \mathrm{mM}$ solution (in DMSO) maintained for about 4 months at room temperature, wrapped in foil, yielded signal intensities comparable to a freshly prepared stock solution (data not shown). We did observe that DPH was susceptible to photobleaching (data not shown) and for this reason is not likely a suitable reagent for confocal microscopy. To minimize the effects of photobleaching in our quantitative analysis, extra wells excluded from the experimental data set were used to establish microscope image acquisition and analysis parameters. DPH is particularly well-suited to high-content imaging, as the automated focus and image acquisition minimizes sample exposure to the imaging light source. Our high-content imaging platform uses an image-based autofocus mechanism, and we used the far-red (e.g., low photobleaching potential) Cy 5 channel to focus.

Assay quality for the HTS context was determined by $\mathrm{Z}^{\prime}$-factor analysis (29), which is a useful calculation that incorporates both assay dynamic range (separation band between positive and negative controls) and assay homogeneity within the respective control groups. In HeLa experiments, we compared the replicates of the OA:BSA to the corresponding BSA-only vehicle control at each $\mathrm{OA}$ concentration. This analysis indicated that both DPH and BODIPY $493 / 503$ reagents typically yielded $\mathrm{Z}^{\prime}$-factors above 0.5 (an excellent HTS assay) at OA concentrations of 63 or 125 $\mu \mathrm{M}$ and higher. At these concentrations, Nile Red was also an effective stain but exhibited lower assay resolution, with
$\mathrm{Z}^{\prime}$-factors between 0.1 and 0.5 (a good HTS assay) even at higher OA concentrations. Nile Red assay resolution did not improve appreciably by using either 20 or $4 \mathrm{ng} / \mathrm{mL}$ of the dye (data not shown). $\mathrm{Z}^{\prime}$-factor analysis of MCF-7 experiments comparing sodium butyrate to mocktreated cells indicated high-content screening assays for cell number, mean total DPH pixel intensity, and phenotypic penetrance (\% DPH bright) to provide suitable assay resolution for HTS above $0.5 \mathrm{mM}$ sodium butyrate and excellent assay resolution above 1 $\mathrm{mM}$ (data not shown).

The cell systems used here provided a useful platform for high-content analysis of LDs, comparing the underutilized dye DPH with other, more common LD stains. The most common of these, Nile Red and BODIPY 493/503, both exhibit substantial FITC fluorescence when LDs are abundant. The addition of a DAPI-range stain for quantitative LD analysis could be very useful for alternative imaging strategies. Here we have demonstrated the efficacy of DPH, validating this stain for high-content screening and HTS applications. DPH exhibited comparable staining patterns to the established LD stains Nile Red and BODIPY 493/503, with similar performance in quantitative analysis of fatty acid dose-response treatment of HeLa cells and butyrate treatment of MCF-7 cells. $Z^{\prime}$-factor analysis of these data reflected that all three reagents could provide good or excellent imagebased HTS assays. DPH costaining with AlexaFluor 488 immunolabeled adipophilin (Figure 2) demonstrated both the LD specificity of DPH and its compatibility with chemical FITC-range fluorophores. Further, the MCF-7 experiments demonstrated DPH compatibility with GFP (Figure 4). The immunofluorescence data also suggests that DPH could be more sensitive than BODPIY 493/503 in detection of very small LDs (Figure 2, B, F, and J). Our data showed that DPH can be used as a total membrane dye, although this may only be practical in the absence of large LDs. The sensitivity, uniformity, unique spectral properties, and extremely low cost of DPH make it a particularly useful reagent for diverse high-throughput imaging and screening applications in lipid biology.

\section{Acknowledgments}

The authors would like to thank Rob Parton, Jon Whitehead, Sandrine Roy,
Ali Naderi, Fiona McMillan, and Sally Martin for their valued input and intellectual support. This work was supported, in part, by grants from the National Health and Medical Research Council (Australia), the University of Queensland, and the UQ Diamantina Institute.

\section{Competing interests}

The authors declare no competing interests.

\section{References}

1. Goodman, J.M. 2009. Demonstrated and inferred metabolism associated with cytosolic lipid droplets. J. Lipid Res. 50:2148-2156.

2. Olofsson, S.O., P. Boström, L. Andersson, M. Rutberg, J. Perman, and J. Borén. 2009 . Lipid droplets as dynamic organelles connecting storage and efflux of lipids. Biochim. Biophys. Acta 1791:448-458.

3. Walther, T.C. and R.V. Farese, Jr. 2009. The life of lipid droplets. Biochim. Biophys. Acta 1791:459-466.

4. Liu, H., J.Y. Liu, X. Wu, and J.T. Zhang. 2010. Biochemistry, molecular biology, and pharmacology of fatty acid synthase, an emerging therapeutic target and diagnosis/ prognosis marker. Int. J. Biochem Mol Biol. 1:69-89.

5. Unger, R.H. and P.E. Scherer. 2010. Gluttony, sloth, and the metabolic syndrome: a roadmap to lipotoxicity. Trends Endocrinol. Metab. 21:345-352.

6. Brookheart, R.T., C.I. Michel, and J.E. Schaffer. 2009. As a matter of fat. Cell Metab. 10:9-12.

7. Martin, S. and R.G. Parton. 2006. Lipid droplets: a unified view of a dynamic organelle. Nat. Rev. Mol. Cell Biol. 7:373378.

8. Conn, H.J. 1977. Biological Stains: A Handbook on the Nature and Uses of the Dyes Employed in the Biological Laboratory, 9th ed. Williams \& Wilkins, Baltimore, MD.

9. Listenberger, L.L. and D.A. Brown. 2007. Fluorescent detection of lipid droplets and associated proteins. Curr. Protoc. Cell Biol. 35:24.2.1-24.2.11.

10. Wolins, N.E., B.K. Quaynor, J.R. Skinner, M.J. Schoenfish, A. Tzekov, and P.E. Bickel. 2005. S3-12, adipophilin, and TIP47, package lipid in adipocytes. J. Biol. Chem. 19:19146-19155.

11. Ashrafi, K., F.Y. Chang, J.L. Watts, A.G. Fraser, R.S. Kamath, J. Ahringer, and G. Ruvkun. 2003. Genome-wide RNAi analysis of Caenorhabditis elegans fat regulatory genes. Nature 421:268-272.

12. Szymanski, K.M., D. Binns, R. Bartz, N.V. Grishin, W.P. Li, A.K. Agarwal, A. Garg, R.G. Anderson, and J.M. Goodman. 2007. The lipodystrophy protein seipin is found at endoplasmic reticulum lipid droplet junctions and is important for droplet 

91:203-215.

Vacuum filtration is one of the most common applications in many laboratories. The new VACUUBRAND ME1 and ME1C diaphragm pumps offer a compact, high performance and easy to use solution for filtration. With flow rates of $13 \mathrm{Ipm}$, ultimate vacuum of 75 Torr and whisper quiet operation these compact pumps offer performance that is far from common.

- Whisper quiet \& low vibration

- Convenient top mounted power switch

- Small footprint requires minimal bench space

- Excellent chemical resistance (ME1C)

- Long diaphragm life

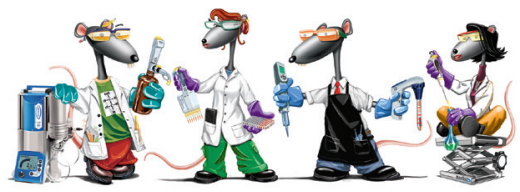

Lab Rats Trust BrandTech!

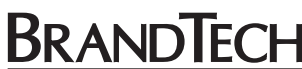
SCIENTIFIC, INC.

Toll Free (888) 522-2726 www.hrandtech.com morphology. Proc. Natl. Acad. Sci. USA 104:20890-20895.

13. Guo, Y., T.C. Walther, M. Rao, N. Stuurman, G. Goshima, K. Terayama, J.S. Wong, R.D. Vale, et al. 2008. Functional genomic screen reveals genes involved in lipid-droplet formation and utilization. Nature 453:657-661

14. Beller, M., C. Sztalryd, N. Southall, M. Bell, H. Jäckle, D.S. Auld, and B. Oliver. 2008. COPI complex is a regulator of lipid homeostasis. PLoS Biol. 6:e282.

15. Grandl, M. and G. Schmitz. 2010 . Fluorescent high-content imaging allows the discrimination and quantitation of E-LDL-induced lipid droplets and Ox-LDLgenerated phospholipidosis in human macrophages. Cytometry A 77:231-242.

16. Whittaker, R., P.A. Loy, E. Sisman, E. Suyama, P. Aza-Blanc, R.S. Ingermanson, J.H. Price, and P.M. McDonough. 2010. Identification of microRNAs that control lipid droplet formation and growth in hepatocytes via high-content screening. J. Biomol. Screen. 15:798-805.

17. Asada, M., A. Rauch, H. Shimizu, H. Maruyama, S. Miyaki, M. Shibamori, H. Kawasome, H. Ishiyama, et al. 2011. DNA binding-dependent glucocorticoid receptor activity promotes adipogenesis via Krüplelike factor 15 gene expression. Lab. Invest.

fluorescent microscopy. Exp. Cell Res. 116:447-450.

28. Brasaemle, D.L., T. Barber, N.E. Wolins, G. Serrero, E.J. Blanchette-Mackie, and C. Londos. 1997. Adipose differentiationrelated protein is an ubiquitously expressed lipid storage droplet-associated protein. J. Lipid Res. 38:2249-2263.

29. Zhang, J.H., T.D. Chung, and K.R. Oldenburg. 1999. A simple statistical parameter for use in evaluation and validation of high throughput screening assays. J. Biomol. Screen. 4:67-73.

30. Liebes, L.F., E. Pelle, D. Zucker-Franklin, and R. Silber. 1981. Comparison of lipid composition and 1,6-diphenyl-1,3,5-hexatriene fluorescence polarization measurements of hairy cells with monocytes and lymphocytes from normal subjects and patients with chronic leukemia. Cancer Res. 41:4050-4056.

31. Smith, P.J., M. Wiltshire, S. Davies, L.H. Patterson, and T. Hoy. 1999. A novel cell permeant and far red-fluorescing DNA probe, DRAQ5, for blood cell discrimination by flow cytometry. J. Immunol. Methods 229:131-139.

32. Guilbaud, N.F., N. Gas, D.A. DuPont, and A. Valette. 1990. Effects of differentiationinducing agents on maturation of human MCF-7 breast cancer cells. J. Cell. Physiol. 145:162-172.

18. Kim, S.J., C. Nian, and C.H.S. McIntosh. 2011. Adipocyte expression of the glucosedependent insulinotropic polypeptide receptor involves gene regulation by $\mathrm{PPAR} \gamma$ and histone acetylation. J. Lipid Res. 52:759770 .

19. Greenspan, P., E.P. Mayer, and S.D. Fowler. 1985. Nile red: a selective fluorescent stain for intracellular lipid droplets. J. Cell Biol. 100:965-973.

20. Greenspan, P. and S.D. Fowler. 1985. Spectrofluorometric studies of the lipid probe, nile red. J. Lipid Res. 26:781-789.

21. Brown, W.J., T.R. Sullivan, and P. Greenspan. 1992. Nile red staining of lysosomal phospholipid inclusions. Histochem. Cell Biol. 97:349-354.

22. Diaz, G., M. Melis, B. Batetta, F. Angius, and A.M. Falchi. 2008. Hydrophobic characterization of intercellular lipids in situ by Nile red red/yellow emission ratio. Micron 39:819-824.

23. Gocze, P.M. and D.A. Freeman. 1994. Factors underlying the variability of lipid droplet fluorescence in MA-10 Leydig tumor cells. Cytometry 17:151-158.

24. Ohsaki, Y., Y. Shinohara, M. Suzuki, and T. Fujimoto. 2010. A pitfall in using BODIPY dyes to label lipid droplets for fluorescence microscopy. Histochem. Cell Biol. 133:477480 .

25. Spandl, J., D.J. White, J. Peychl, and C. Thiele. 2009. Live cell multicolor imaging of lipid droplets with a new dye, LD540. Traffic 10:1579-1584.

26. Shinitzky, M. and Y. Barenholz. 1974. Dynamics of the hydrocarbon layer in liposomes of lecithin and sphingomyelin containing dicetylphosphate. J. Biol. Chem. 249:2652-2657.

27. Collard, J.G. and A. De Wildt. 1978. Localization of the lipid probe 1,6-diphenyl$1,3,5$ hexatriene $(\mathrm{DPH})$ in intact cells by
33. Davis, T., C. Kennedy, Y.E. Chiew, C.L. Clarke, and A. deFazio. 2000. Histone deacetylase inhibitors decrease proliferation and modulate cell cycle gene expression in normal mammary epithelial cells. Clin. Cancer Res. 6:4334-4342.

34. Abe, M. and D.W. Kufe. 1984. Effect of sodium butyrate on human breast carcinoma (MCF-7) cellular proliferation, morphology, and CEA production. Breast Cancer Res. Treat. 4:269-274.

Received 6 November 2010; accepted 16 May 2011.

Address correspondence to Thomas J. Gonda, University of Queensland Diamantina Institute, Princess Alexandra Hospital, Ipswich Rd, Brisbane QLD 4102, Australia. email: t.gonda@uq.edu.au

To purchase reprints of this article, contact: biotechniques@fosterprinting.com 